\title{
Il rifiuto del trattamento sanitario da parte dei genitori della persona minore di età e la prevenzione del ricorso al giudice tutelare: il caso della trasfusione ematica
}

\author{
Matteo Bolcato, Daniele Rodriguez, Anna Aprile \\ Medicina Legale, Università degli Studi di Padova
}

\begin{abstract}
Nella pratica clinica possono verificarsi situazioni di contrasto tra genitori e medici in merito alle scelte di cura per i figli minorenni. Un caso relativamente frequente è rappresentato dal mancato consenso alla trasfusione ematica da parte dei genitori. Larticolo delinea un aggiornato inquadramento legislativo sulla questione e un possibile approccio operativo, che nella pratica si è rivelato efficace al fine di soddisfare le esigenze di salute dei pazienti e le volontà espresse dai genitori.
\end{abstract}

\section{In clinical practice, there may be situations of conflict between parents and doctors regarding care choices for minors. A relatively frequent case is represented by the parents' refusal to consent to blood transfusion. The article outlines an updated legislative framework on the issue and a possible operational approach, which has proved to be effective in meeting the health needs of patients and the wishes expressed by parents.}

\section{Introduzione}

Nella pratica clinica con le persone minori di età possono verificarsi situazioni di contrasto tra genitori e medici in merito alle scelte e ai provvedimenti di cura. Uno dei casi frequenti è rappresentato dal mancato consenso, da parte dei genitori, alla trasfusione di emocomponenti proposta dal medico per i loro figli. Ciò può suscitare preoccupazione nei medici che considerano inappropriata e incongrua tale scelta dei genitori.

Nel presente articolo delineiamo un aggiornato inquadramento legislativo sulla questione e suggeriamo un possibile approccio operativo che, nella nostra esperienza, si è rivelato efficace al fine di soddisfare le esigenze di salute dei pazienti tenendo conto delle volontà espresse dai genitori.

\section{Inquadramento legislativo}

su informazione alle persone minori di età e consenso o rifiuto dei genitori

La legge 22 dicembre 2017, n. 219 "Norme in tema di consenso informato e direttive anticipate di trattamento" [1] dedica i primi due commi e l'ultimo comma dell'articolo 3 alle persone minori di età:

"1. La persona minore di età (...) ha diritto alla valorizzazione delle proprie capacità di comprensione e di decisione, nel rispetto dei diritti di cui all'articolo 1 , comma 1.
Deve ricevere informazioni sulle scelte relative alla propria salute in modo consono alle sue capacità per essere messa nelle condizioni di esprimere la sua volontà.

2. Il consenso informato al trattamento sanitario del minore è espresso o rifiutato dagli esercenti la responsabilità genitoriale o dal tutore tenendo conto della volontà della persona minore, in relazione alla sua età e al suo grado di maturità, e avendo come scopo la tutela della salute psicofisica e della vita del minore nel pieno rispetto della sua dignità. (...)

5. Nel caso in cui (...) il rappresentante legale della persona minore rifiuti le $\mathrm{cu}^{-}$ re proposte e il medico ritenga invece che queste siano appropriate e necessarie, la decisione è rimessa al giudice tutelare su ricorso del rappresentante legale della persona interessata o dei soggetti di cui agli articoli 406 e seguenti del codice civile o del medico o del rappresentante legale della struttura sanitaria."

In sintesi, il comma 1 è dedicato alla informazione alla persona minore di età ed alla sua comprensione; il comma 2 riguarda l'espressione del consenso o il rifiuto da parte dei genitori; il comma 5 tratta del ricorso al giudice tutelare.

Il comma 1 sancisce il diritto della persona minore di età alla valorizzazione delle sue capacità di comprensione e di decisione; di conseguenza, questa persona riceve dal medico le informazioni necessarie a formarsi un'opinione, e quindi esprime una volontà sulle scelte relative alla propria salute in modo consono alle proprie capacità. Il comma 2, pur conferendo ai genitori la potestà di esprimere il consenso o il rifiuto, stabilisce che essi, avendo come scopo la tutela della salute e della vita della persona minore, devono tener conto della sua volontà, ovviamente in relazione alla sua età e al suo grado di maturità.

È pacifico, pur se non espressamente previsto, che il medico proceda a informare anche i genitori con un linguaggio consono a loro e diverso da quello adottato con la persona minore. In sostanza, questi due commi descrivono una relazione di cura fra più persone che comunicano reciprocamente e partecipano al processo decisionale, la formulazione conclusiva del quale è affidata ai genitori. Pare razionale che la informazione del medico alla persona minore di età avvenga in presenza dei genitori e che i genitori manifestino il consenso o il rifiuto in sua presenza.

È opportuno il richiamo del comma 1 alle capacità di comprensione e di decisione della persona e del comma 2 alla sua età e al suo grado di maturità; la procedura proposta è impensabile nel caso che si tratti di un bambino molto piccolo ma assume connotazioni di sempre maggior concretezza e fattibilità con il progredire dell'età e, soprattutto, della maturità.

Il comma 5 prospetta il caso che i genitori rifiutino le cure proposte dal medico che le ritiene appropriate e necessarie; in tal caso, è prevista la possibilità del ricorso al giudice tutelare da parte di vari soggetti, fra i quali sono contemplati il medico e il rappresentante legale della struttura sanitaria. Può non essere fuori luogo, comunque, di fronte a un rifiuto dei genitori, che il medico verifichi preliminarmente se esso sia derivato da un difetto di comprensione dei genitori ed è ovvio che in tal caso 
egli debba impegnarsi in una più efficace informazione.

In caso di persistenza nel rifiuto da parte dei genitori, il comma 5 dell'articolo 3 individua chiaramente l'organo giudiziario a cui il medico potrà, e a nostro avviso dovrà, rivolgersi per dirimere la questione, ossia il giudice tutelare. Riteniamo che sia dovere e non semplice facoltà del medico ricorrere al giudice tutelare, stante la peculiarità della posizione di garanzia che egli riveste nei confronti della persona minore di età nella prospettiva che il rifiuto dei genitori porti a conseguenze nefaste.

È da ribadire che la legge n. 219 identifica nel giudice tutelare l'organo giudiziario competente. Questa nozione non è evidentemente di patrimonio comune, dato che in alcuni casi di rifiuto di trasfusione i curanti hanno contattato la procura della Repubblica o il tribunale per i minorenni. Tale percorso improprio ha spesso portato a uno scontro aperto con i genitori, risoltosi solo sul piano legale.

Un caso giudiziario che ha ricevuto attenzione mediatica è avvenuto nel settembre 2019 in un ospedale lombardo, quando una coppia ha portato al pronto soccorso di un ospedale una bambina di 10 mesi che, dopo una caduta, aveva sviluppato un ematoma extradurale. I curanti si spesero in modo tempestivo per garantirle le migliori cure attuando l'intervento neurochirugico necessario. I genitori acconsentirono a prestare il consenso per effettuare tutte le cure ritenute dai medici necessarie e appropriate a esclusione della somministrazione di emocomponenti (globuli rossi, piastrine e plasma), accettando invece emoderivati (frazioni più piccole del sangue come l'albumina, i fattori della coagulazione, le gammaglobuline, il fibrinogeno, ecc.), per motivazioni religiose. A seguito di tale rifiuto i curanti sono ricorsi al tribunale per i minorenni di Milano, che ha disposto la limitazione della responsabilità genitoriale e affidato la persona minore di età, in ordine alle scelte sanitarie, al legale rappresentante dell'ospedale. Dopo l'esecuzione dell'intervento chirurgico, la piccola è stata dimessa in buone condizioni e non è mai stata necessaria l'esecuzione di emotrasfusioni. I genitori hanno ricorso contro il provvedimento del tribunale e la corte d'appello di Milano si è pronunciata con decreto n. 1991 del 2020 nell'udienza del 10 settembre 2020 stabilendo che, dal punto di vista legale, la procedura prevista dalla legge non era stata eseguita e che è il giudice tutelare l'organo deputato a decidere le controversie in caso di dissenso fra genitori e medici; pertanto, in accoglimento del reclamo, era disposta la revoca del decreto emesso.
In definitiva la corte d'appello di Milano ha stabilito che l'impianto legale indicato dalla legge n. 219 del 2017 è l'unico che deve essere seguito nei casi di rifiuto di $\mathrm{cu}^{-}$ re o trattamenti da parte dei genitori e che l'organo decisore su questo tema è il giudice tutelare; inoltre ha sottolineato che il mero rifiuto delle emotrasfusioni in aderenza a un credo religioso non può essere la base per valutare i genitori non idonei a occuparsi di un bambino. Altre tre corti d'appello si sono pronunciate nell'ultimo anno su casi simili adottando la stessa indicazione generale (Roma n. 772 del 2019, Catania n. 205 del 2020, Perugia n. 763 del 2020). Le previsioni del comma 5 dell'articolo 3 vanno tuttavia considerate alla luce del comma 7 dell'articolo 1 della legge n. 219.

"7. Nelle situazioni di emergenza o di urgenza il medico e i componenti dell'équipe sanitaria assicurano le cure necessarie, nel rispetto della volontà del paziente ove le sue condizioni cliniche e le circostanze consentano di recepirla".

Ciò in pratica significa che, in caso di rifiuto dei genitori di interventi assolutamente necessari, occorre valutare se l'intervento rifiutato è differibile: in caso affermativo il medico procederà al ricorso al giudice tutelare.

In caso contrario, se esiste cioè "urgenza", il medico procederà a svolgere gli atti non differibili, senza necessità di preventiva decisione del giudice tutelare. Tale sarà la condotta del medico, anche qualora l'urgenza subentri nell'intervallo di tempo che precede la decisione del giudice tutelare al quale è già stato fatto ricorso.

Va da sé che la valutazione dell'urgenza e le caratteristiche dell'intervento debbano basarsi su elementi oggettivi ed essere conformi alle raccomandazioni delle linee guida e, in mancanza, delle buone pratiche clinico-assistenziali. Riteniamo opportuno che il medico dia comunicazione al giudice tutelare dei casi in cui abbia proceduto a interventi di urgenza in presenza di rifiuto dei genitori.

La relazione di cura presuppone lealtà nella comunicazione. Pertanto il quadro legislativo generale, che in primo luogo deve essere chiaro ai medici curanti, va da loro illustrato ai genitori che potrebbero non essere pienamente consapevoli dei limiti previsti dalla legge all'esercizio della responsabilità genitoriale qualora questa possa confliggere con l'interesse del figlio minore di età.

La legge n. 219, al suo articolo 1, comma 8 stabilisce che "Il tempo della comunicazione tra medico e paziente costituisce tempo di cura". Il tempo dedicato a parlare e confrontarsi con i genitori e i loro figli minori di età è prezioso al fine di favorire la consapevolezza e la responsabilizzazione nelle decisioni e di evitare contrasti basati sulle incomprensioni e spiacevoli ricorsi all'autorità giudiziaria.

\section{Le possibilità cliniche}

Tali disposizioni hanno ovviamente valore anche in caso di rifiuto opposto dai genitori a una trasfusione ematica al loro figlio, ritenuta opportuna dal medico curante.

È importante considerare che il ricorso al giudice tutelare, pur consentendo in genere la soluzione del caso concreto:

a può determinare criticità nella relazione fra figlio e genitori, legate alla eventuale percezione da parte del primo, di una inadeguatezza di questi ultimi, essendo stata riconosciuta da un giudice, almeno implicitamente, la loro incompetenza a tutelare al meglio la prole nel momento del bisogno;

b sancisce comunque il fallimento della relazione di cura, con conseguenti difficoltà a garantire nel futuro la migliore qualità delle cure, in particolare nel caso di pazienti affetti da patologie croniche.

Nella pratica della medicina legale clinica [2] è nostro obiettivo evitare involuzioni della relazione di cura, favorendo la consapevolezza di tutte le persone che a essa partecipano, le modalità della comunicazione e il contenuto della informazione, attraverso queste attività:

- promozione delle conoscenze dei medici circa le corrette indicazioni alla trasfusione;

- accuratezza del processo di informazione nei confronti dei genitori;

- promozione di un processo di alleanza tramite la pianificazione condivisa delle cure.

\section{La promozione delle conoscenze dei medici circa le corrette indicazioni alla trasfusione}

Negli ultimi anni si è svolta un'imponente attività di ricerca scientifica sui risultati attesi e reali della terapia trasfusionale e sulle possibilità di una sua riduzione o eliminazione, al fine di proteggere i pazienti dai rischi trasfusionali e migliorarne le condizioni di salute oltre a risparmiare emocomponenti in una situazione generale di risorse ematiche progressivamente più limitate [3-5]. L'Organizzazione Mondiale della Sanità fin dal 2010 ha introdotto una risoluzione (World Health Assembly WHA 63.12 resolution on "Availability, safety and quality of blood products" del 21 maggio 2010) per la creazione e l'implementazione del programma internazionale Patient 
Blood Management (PBM) che può essere definito come: "Approccio multidisciplinare, multimodale e personalizzato volto a ridurre o eliminare la necessità di trasfusioni allogeniche attraverso la gestione evidence-based dell'anemia, la riduzione delle perdite e l'ottimizzazione delle strategie volte al risparmio del sangue."

Nella letteratura scientifica internazionale sono diversi i contributi che mostrano l'esecuzione di interventi complessi e il trattamento di malattie gravi senza l'utilizzo di sangue, con risultati simili, se non migliori, a quelli dei pazienti trasfusi; questi interventi includono procedure complesse come i trapianti di organi e la chirurgia a cuore aperto, anche nei bambini e nei neonati [5-15]. Questo approccio, non solo utile a chi rifiuta il sangue, diviene anche prezioso patrimonio culturale per la cura di tutti i pazienti in sicurezza $[4,16]$. Si è quindi delineato un percorso atto a contrastare l'anemia e prevenire le perdite ematiche durante e dopo gli interventi chirurgici. Il percorso è caratterizzato da oltre 100 strumenti (farmacologici e non) finalizzati a questo obiettivo [17]; in questo percorso la trasfusione ematica diventa un'opportunità da conside- rare extrema ratio, da attuare solo dopo aver esaurito tutte le precedenti strategie.

L'evoluzione di queste conoscenze è stata relativamente rapida e ha interessato un tipo di terapia che "tradizionalmente" è stato di automatica e indefettibile applicazione in caso di anemizzazione di una certa entità. La formazione universitaria dei medici e la loro educazione continua non sempre sono state conformi al cambiamento delle conoscenze e in particolare al programma internazionale PBM. Ne consegue che i programmi di insegnamento a tutti i livelli, di base e post base, devono dedicare a questo argomento specifica attenzione. In ogni caso, è necessario che i medici acquisiscano, anche con formazione autogestita, una competenza specifica in materia di indicazioni e controindicazioni alla terapia trasfusionale e si mantengano permanentemente aggiornati.

L'accuratezza del processo di informazione nei confronti dei genitori

La competenza dei medici in materia di trasfusioni è presupposto indispensabile perché essi possano operare strategie terapeutiche adeguate e coerenti con le esigen- ze contingenti della persona, con l'utilizzo congruo della risorsa sangue e con il bilanciamento fra i benefici attesi e i rischi connessi a questa terapia. I medici dovranno saper esprimere questa competenza nelle informazioni da fornire nell'ambito della relazione di cura.

La relazione di cura è basata sulla comunicazione. La comunicazione, per quanto riguarda il medico, è chiamata a veicolare contenuti adeguati a dare risposta ai bisogni globali della persona e deve avere anche un contenuto tecnico per permettere alla persona di verificare, per quanto possibile, l'adeguatezza del progetto terapeutico che viene proposto dal medico alle buone pratiche basate sulle evidenze scientifiche disponibili [16]. In particolare, il medico deve dare assicurazione che la proposta di ricorrere a trasfusione ematica è rispettosa delle strategie del PBM.

\section{La promozione di un processo di alleanza tramite la pianificazione condivisa delle cure}

La legge n. 219 introduce l'istituto della pianificazione condivisa delle cure [2], specificamente disciplinata dall'articolo 5 .

BOX 1. Esempio di pianificazione condivisa delle cure con genitori che rifiutano la trasfusione ematica e figlio minore di età

II giorno [...] ha avuto luogo un incontro tra il paziente [...] di anni 12, i genitori e i medici curanti [...] [pediatri]. Ha partecipato all'incontro altresì il dr. [...] medico legale. Scopo dell'incontro era definire una pianificazione condivisa del percorso terapeutico del paziente, affetto da Sarcoma di Ewing [si descrive la condizione patologica e l'iter terapeutico adiuvante prospettato]. II paziente mostra di avere conoscenza della propria malattia e di avere compreso l'iter terapeutico proposto. Egli esprime ai medici curanti la volontà di non essere sottoposto a terapia trasfusionale per motivi religiosi in quanto Testimone di Geova, in pieno accordo con i suoi genitori.

I medici curanti, che già hanno precedentemente comunicato al paziente e ai genitori le informazioni relative alle indicazioni e alle possibili complicanze del trattamento chemioterapico, ritornano sulla questione relativa al rifiuto trasfusionale precisando che la terapia è gravata da un rischio di anemia anche severa, con necessità di trasfusione di eritrociti e/o piastrine nella maggior parte dei casi consimili.

I genitori del paziente chiedono di intervenire sull'anemia con terapie di supporto e farmaci per prevenirla confermando il loro rifiuto alla trasfusione ematica. II dr. [medico curante], nell'assecondare questa richiesta, prospetta la possibilità di utilizzo di strategie atte alla prevenzione e cura dell'anemia (es. eritropoietina, ferro, complessi vitaminici, riduzione del numero di prelievi, ecc.), precisando inoltre come anche alcune di queste possano essere foriere di possibili complicanze.

I genitori e il giovane paziente, dimostrano di aver ben compreso le informazioni ricevute, sia in relazione alle caratteristiche della terapia sia in relazione al rischio per la salute e per la vita derivante da una anemizzazione severa non trattata con trasfusioni ematiche ed esprimono il consenso all'utilizzo di terapie di supporto nonché di albumina e fattori della coagulazione negandolo per la trasfusione ematica.

II medico legale comunica ai genitori e al paziente che, pure nel pieno rispetto delle scelte individuali e di chi esercita la responsabilità genitoriale, in caso di persona minorenne il dovere giuridico e deontologico del medico è quello di ricorrere al giudice tutelare in caso di rifiuto di cure ritenute appropriate e necessarie dai curanti procedendo comunque tempestivamente alle cure indispensabili e indifferibili (articolo 3, comma 5, della legge 219/2017; articolo 37 codice di deontologia medica).

Si illustra ai genitori e al paziente, pertanto, quello che sarà l'iter al quale i medici curanti si atterranno nella cura del paziente in relazione alla trasfusione ematica:

1) fino al momento in cui la condizione clinica lo consente, i medici curanti si atterranno alla richiesta del paziente e dei genitori di non effettuare trasfusioni ematiche e adotteranno tutte le necessarie strategie al fine di evitare la trasfusione allogenica;

2) qualora la condizione clinica evolvesse verso situazioni di criticità risolvibili soltanto con la trasfusione, permanendo il rifiuto dei genitori al trattamento, si farà ricorso al giudice tutelare, riferendo che il rappresentante legale della persona minore di età rifiuta un trattamento che il medico curante ritiene appropriato e necessario.

Qualora nel periodo di tempo intercorrente, necessario all'istruzione della pratica e delibera del giudice tutelare, dovesse presentarsi un'emergenza/urgenza risolvibile unicamente per mezzo di terapia emotrasfusionale, questa verrà somministrata in conformità alla posizione di garanzia in capo ai medici curanti.

I genitori riferiscono di avere piena fiducia nei medici curanti e di aver ben compreso tutte le informazioni fornite, in particolare l'obbligo di rimettere la decisione al giudice tutelare e la necessità di eventuale somministrazione di terapia emotrasfusionale al figlio, qualora si dovessero presentare situazioni di emergenza/urgenza tali da richiedere trasfusione indifferibile.

Data, (Firme dei genitori, del figlio e dei medici presenti) 
"1. Nella relazione tra paziente e medico di cui all'articolo 1, comma 2, rispetto all'evolversi delle conseguenze di una patologia cronica e invalidante o caratterizzata $\mathrm{da}$ inarrestabile evoluzione con prognosi infausta, può essere realizzata una pianificazione delle cure condivisa tra il paziente e il medico, alla quale il medico e l'équipe sanitaria sono tenuti ad attenersi qualora il paziente venga a trovarsi nella condizione di non poter esprimere il proprio consenso o in una condizione di incapacità”.

La pianificazione condivisa delle cure si pone nel contesto della "relazione di cura e di fiducia tra paziente e medico", stante il rinvio che il comma citato fa al comma 2 dell'articolo 1. Riteniamo che essa, pur se non contemplata come obbligatoria nella legge n. 219, collocandosi nell'ambito di detta relazione, qualora non sia richiesta dal paziente, vada comunque attivamente ricercata dal medico e proposta al paziente, reso consapevole dal medico stesso.

La pianificazione condivisa delle cure rientra dunque nella "relazione di cura": perciò è espressione da un lato delle aspirazioni di cura della persona, dall'altro della responsabilità del medico, chiamato a impegnarsi per rendere concreto il diritto del paziente a essere reso consapevole e a esprimere le proprie aspirazioni e i propri desideri.

Lo svolgimento pratico si articola su uno o più incontri, ciascuno dei quali comporta una fase preliminare di carattere informativo, una fase intermedia di valutazione delle indicazioni fornite dal medico e delle aspirazioni espresse dal paziente e una fase finale di accordo tra i partecipanti. Tale processo è formalizzato in un apposito verbale, firmato da tutte le parti, che è inserito nella cartella clinica e nel fascicolo sanitario elettronico del paziente. È consigliabile fornire copia del verbale al paziente stesso e al fiduciario, se nominato.

Per quanto riguarda i pazienti pediatrici, la pianificazione condivisa delle cure è il contesto ideale di confronto fra le persone che rientrano nella relazione di cura, vale a dire il medico, i genitori e il bambino (ovviamente, quando quest'ultimo abbia sviluppato la maturità necessaria). In ambito pediatrico può essere uno strumento decisivo per il perseguimento della personalizzazione della medicina, richiamando l'attenzione del medico sulle esigenze e sulla biografia del singolo malato, al quale sono fornite informazioni adeguate alla sua capacità di comprensione e che possono essere eventualmente precisate in termini tecnicamente più precisi, comunque comprensibili, ai genitori.

Nella nostra esperienza, l'adozione di questo strumento con i pazienti minori di età si è rivelato particolarmente utile per risolvere episodi che avrebbero potuto sfociare in situazioni conflittuali con i genitori che rifiutavano la somministrazione trasfusionale per i figli. In questi casi, l'attenzione che i medici hanno dimostrato al programma del PBM e le garanzie che sono state fornite circa la sua rigorosa applicazione e il ricorso alla trasfusione ematica soltanto come extrema ratio nel percorso clinico terapeutico [19] si sono rivelate fondamentali per costruire una solida alleanza con la persona minore di età e con i suoi genitori.

Questo tipo di approccio ha permesso in molti casi di evitare il ricorso al giudice tutelare.

Nel Box 1 è riportato un esempio di verbale di pianificazione condivisa delle cure.

\section{Il ruolo del clinical risk management}

Il clinical risk management è abitualmente identificato come un complesso di sistemi organizzati, finalizzati a migliorare la qualità delle prestazioni sanitarie e a garantire la sicurezza dei pazienti, dei visitatori e dei dipendenti tramite la identificazione, la valutazione e la limitazione dei rischi [19-21]. Il tema in discussione riveste interesse nell'ambito del clinical risk management, perché uno dei fattori dai quali può derivare il rifiuto dei genitori può essere costituito dalla incompetenza del medico nel gestire la relazione di cura con persone che esprimono valori, esigenze e/o convincimenti estranei alla cultura del medico e perché, come già esposto, il ricorso al giudice tutelare compromette la relazione di cura e può incrinare la relazione dei figli verso i genitori.

La nostra tesi è che, nell'ambito del clinical risk management, vadano prese in considerazione:

1 la prevenzione del ricorso al giudice tutelare ponendo attenzione alla relazione di cura, con riferimento a modalità, stile e contenuti della comunicazione al fine di evitare pertinaci rifiuti dei genitori a trattamenti indicati;

2 la costruzione, in caso di ineludibile ricorso al giudice tutelare, di due sistemi volti rispettivamente (il primo) alla tutela delle persone minori di età che vivono l'esperienza del confronto con genitori ai quali è stata temporaneamente rimossa la potestà e (il secondo) alla ricostruzione della relazione di cura compromessa dal ricorso al giudice tutelare.

La prima area di intervento, quella della prevenzione del ricorso al giudice tutelare, si basa su: individuazione delle relazioni di cura difficili; analisi dei fattori che determinano le difficoltà, con particolare attenzione alla competenza dei medici di partecipare alla relazione; trattamento di questi fattori; monitoraggio.

Circa la seconda area di intervento proposta, il primo sistema si fonda sulla ipotesi del rischio che il ricorso al giudice tutelare incrini la relazione del figlio con il genitore; esso comporta comunque, in tempi spesso brevissimi, l'analisi della densità del rischio nel caso concreto e la elaborazione di strategie specifiche, nei tempi concessi dalla situazione, dirette alla preparazione della persona minore di età all'evento traumatico della rimozione della potestà dei genitori.

Il secondo sistema prospettato, quello finalizzato alla ricostruzione della relazione di cura, è oggettivamente il più problematico e non si presta a schemi di soluzioni preconfezionate. Il rischio è in re ipsa ed è costituito dalla impossibilità di mantenere la relazione di cura, che agli occhi dei genitori è stravolta unilateralmente a causa del ricorso del medico al giudice tutelare ed è divenuta difficile per la persona minore di età, potenzialmente disorientata di fronte alla dura contrapposizione dei diversi convincimenti dei genitori e del medico. Una analisi adeguata può essere sviluppata con lo strumento dell'audit clinico.

Il ricorso al giudice tutelare è visto spesso, emotivamente, come punto di arrivo, come chiave di soluzione di un problema; in realtà il ricorso costituisce esso stesso un problema, nuovo, da affrontare ed è comunque il sigillo del fallimento della relazione di cura.

\section{Conclusioni}

I casi di rifiuto del trattamento sanitario - trasfusioni ematiche incluse - da parte dei genitori della persona minore di età comportano una gestione complessa basata sulle procedure schematizzate nella Figura 1. All'interno della relazione di cura vanno attivate le risorse di tutti i partecipanti al fine di raggiungere una soluzione e l'intervento del giudice tutelare corrisponde al fallimento della cura.

E necessario che le questioni siano affrontate da parte di professionisti competenti con mezzi razionali e organizzati [16].

Circa i mezzi, da un lato si è rivelata utile l'adozione della pianificazione condivisa delle cure [22], che merita quindi di essere ampiamente diffusa in ambito pediatrico più di quanto lo sia attualmente, dall'altro lato è raccomandabile una strategia di clinical risk management volta a prevenire i rischi del ricorso al giudice tutelare e alla loro gestione nel caso che tale ricorso sia ineludibile.

Per quanto riguarda i medici, è necessario che la formazione universitaria garantisca 


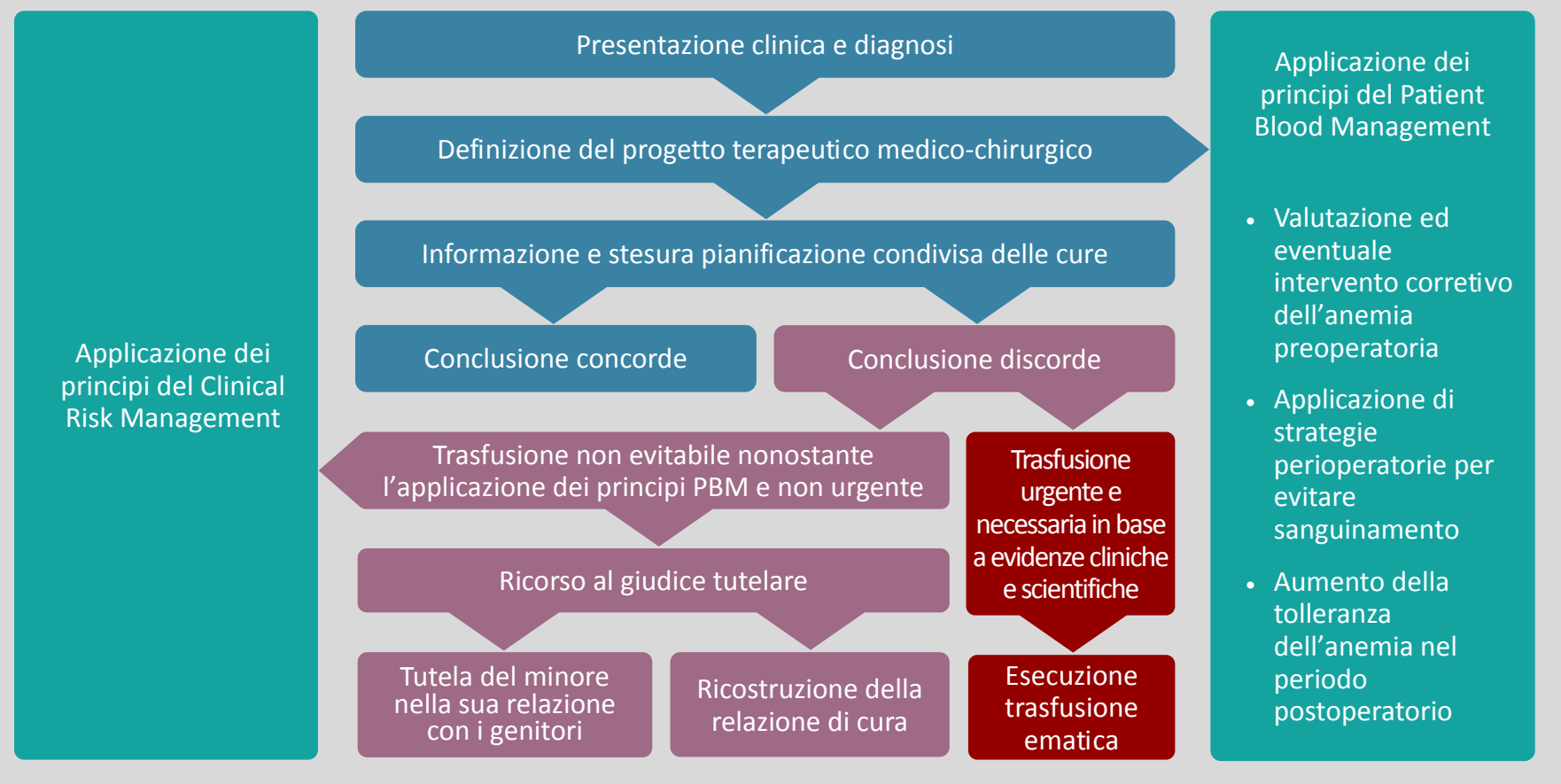

Figura 1. II processo decisionale nell'ambito della relazione fra medico, minore di età e suoi genitori.

il raggiungimento di elevate abilità nella relazione di cura con la persona minore di età e con i suoi genitori, soprattutto quando questi manifestino il loro rifiuto ad alcuni specifici trattamenti e che, successivamente, il professionista mantenga e, se possibile, perfezioni dette abilità.

\section{$\bowtie$ matteobolcato@gmail.com}

1. Di Paolo M, Gori F, Papi L, Turillazzi E. A review and analysis of new Italian law 219/2017: "provisions for informed consent and advance directives treatment". BMC Med Ethics. 2019 Mar 4;20(1):17.

2. Bolcato M, Fassina G, Sanavio M, Aprile A. The new Italian law 219/2017: an extraordinary clinical tool in internal medicine. Italian Journal of Medicine 2020;3:184-8. https://doi. org/10.4081/itjm.2020.1280.

3. Bolcato M, Rodriguez D, Aprile A. Patient Blood Management: un approccio possibile all'anemia anche nei pazienti pediatrici. Quaderni acp 2020;6:271-3.

4. Bolcato M, Russo M, Rodriguez D, Aprile A. Patient blood management implementation in light of new Italian laws on patient's safety. Transfus Apher Sci. 2020 Aug;59(4):102811.

5. Leahy MF, Hofmann A, Towler S, et al. Improved outcomes and reduced costs associated with a health-system-wide patient blood management program: a retrospective observational study in four major adult tertiary-care hospitals. Transfusion. 2017 Jun;57(6):134758.

6. Hare GM, Freedman J, Mazer DC. Review article: risks of anemia and related man- agement strategies: can perioperative blood management improve patient safety? Can J Anaesth. 2013 Feb;60(2):168-75.

7. Langhi DM, Covas DT, Marques JFC, et al. Guidelines on transfusion of red blood cells: Prognosis of patients who decline blood transfusions. Hematol Transfus Cell Ther. Oct-Dec 2018;40(4):377-81.

8. Bhaskar B, Jack RK, Mullany D, Fraser J. Comparison of outcome in Jehovah's Witness patients in cardiac surgery: an Australian experience. Heart Lung Circ. 2010 Nov;19(11):655-9.

9. Posluszny JA, Napolitano LM. How do we treat life-threatening anemia in a Jehovah's Witness patient? Transfusion. 2014 Dec;54(12):3026-34.

10. Vaislic CD, Dalibon N, Ponzio O, et al. Outcomes in cardiac surgery in 500 consecutive Jehovah's Witness patients: 21 year Experience. J Cardiothorac Surg. 2012 Sep 27;7:95.

11. Darwish A. Liver transplant in Jehovah's Witnesses patients. Curr Opin Organ Transplant. 2011 Jun;16(3):326-30.

12. Mazza P, Palazzo G, Minoia C, et al. Autologous and allogeneic stem cell transplant in Jehovah's Witnesses: A single-center experience on 22 patients. Bone Marrow Transplant. $2016 \mathrm{Jul} ; 51(7): 1002-3$.

13. Ford PA, Grant SJ, Mick R, Keck G. Autologous Stem-Cell Transplantation Without Hematopoietic Support for the Treatment of Hematologic Malignancies in Jehovah's Witnesses. J Clin Oncol. 2015 May 20;33(15):1674-9.

14. Jabbour N, Gagandeep S, Mateo R, et al. Transfusion free surgery: Single institution experience of 27 consecutive liver transplants in Jehovah's witnesses. J Am Coll Surg. 2005 Sep;201(3):412-7.

15. Partovi S, Bruckner BA, Staub D, et al. Bloodless lung transplantation in Jehovah's Witnesses: Impact on perioperative parameters and outcome compared with a matched control group. Transplant Proc. Jan-Feb 2013;45(1):335-41.

16. Bolcato M, Russo M, Trentino K, et al. Patient blood management: The best approach to transfusion medicine risk management. Transfus Apher Sci. 2020 Aug;59(4):102779.

17. Meybohm P, Richards T, Isbister J, et al. Patient Blood Management Bundles to Facilitate Implementation. Transfus Med Rev. 2017 Jan;31(1):62-71.

18. Bolcato M, De Salvia A, Rodriguez D, Aprile A. Is the Italian consent to transfusion really informed? A medico-legal analysis between old ghosts and new evidence. Transfus Apher Sci. 2020 Oct;59(5):102823.

19. Bolcato M, Fassina G, Rodriguez D, et al. The contribution of legal medicine in clinical risk management. BMC Health Serv Res. 2019 Feb 1;19(1):85.

20. Bolcato M, Fassina G, Sanavio M, Aprile A. Seventeen-year medical professional liability experience in a level III university hospital. Curr Pharm Biotechnol. 2020 Oct 15.

21. Rodriguez D, Arseni A. Clinical governance e clinical risk management. In: Rodotà S, Zatti P (a cura di). Trattato di Biodiritto. La responsabilità in Medicina. Giuffrè Editore, 2011:85-111.

22. Bevilacqua G, Bolcato M, Rodriguez D, Aprile A. Shared care plan: an extraordinary tool for the personalization of medicine and respect for self-determination. Acta Biomed. 2020 Apr 29;92(1):e2021001. 